\title{
Relation between quality of life and physical literacy of young adolescents with Autism Spectrum Disorder
}

\author{
Iuliia Pavlova ${ }^{1}$, Ivanna Bodnar ${ }^{1}$, Ali Hamade ${ }^{1,2}$, Dariusz Mosler ${ }^{3}$, \\ Eligiusz Małolepszy ${ }^{3}$ and Teresa Drozdek-Małolepsza ${ }^{3}$ \\ ${ }^{1}$ Theory and Methods of Physical Culture Department, L'viv State University of Physical Culture, Ukraine \\ 2 Shafallah Center, Qatar \\ ${ }^{3}$ Department of Kinesiology and Health Prevention, Jan Dlugosz University in Czestochowa, Poland
}

Pavlova, I., Bodnar, I., Hamade,

A., Mosler, D., Małolepszy, E., Drozdek-Małolepsza, T. (2021).

Relation between quality of life and physical literacy

of young adolescents

with Autism Spectrum Disorder.

International Journal

of Special Education, 36(2), 13-26

CORRESPONDING AUTHOR:

Dariusz Mosler; d.mosler.@ujd.edu.pl

DOI:

https://doi.org/10.52291/ijse.2021.36.14

COPYRIGHT STATEMENT:

Copyright: (C) 2021 Authors.

Open access publication under the terms and conditions

of the Creative Commons

Attribution (CC BY)

license (http://creativecommons.

org/licenses/by/4.0/).

\begin{abstract}
:
This study aims to obtain knowledge of how ASD individuals differ from their normally developed equals in terms of physical literacy and quality of life on a stage of early adolescents. Data have been collected from two samples: 18 males with autism spectrum disorders (ASD) aged 11-13 years (main group) and 30 males aged 11-12 years without ASD diagnosis (control group, CG). Canadian Assessment of Physical Literacy - second edition (CAPL-2) has been applied for assessing the level of physical literacy. Pediatric Quality of Life Inventory (Peds $\mathrm{QL}^{\mathrm{Tm}} 4.0$ ) has been used to assess the quality of life. Children with ASD have the most significant lag in static strength endurance of muscles (84\%), coordination, dynamic, and static balance lag behind the standard (by $67 \%$ to $45 \%$ ). The quality of life following the Emotional Functioning scale is relatively low both in children with ASD $(58.42 \pm$ 19.51 points) and healthy respondents ( $69.50 \pm 17.04$ points). Respondents with ASD indicate that it is hard to establish good relationships with other children (47.37\%), had complaints about bullying (21.05\%), and maintain the required pace of the game (68.43\%). Physical education programs should focus more on coping with disabled children in the integration environment and better preparation of ASD children for physical activity which requires social interaction like playing sports games.
\end{abstract}

Keywords: motherhood; family; profound intellectual disability; adulthood. 


\section{INTRODUCTION}

Autism spectrum disorder (ASD) is still not fully understood a wide range of neurodevelopmental disorders (autism, Asperger syndrome, Rett's disorder, childhood disintegrative disorder, pervasive developmental disorder - not other specified) (Sahin \& Sur, 2015) that characterized by impairments in social deficit and communication. Its etiology is very complex; the number of environmental and genetic factors is still increasing with new studies (Newschaffer et al., 2007). Both genetic dependencies and environmentally induced epigenetic changes can cause ASDs (Persico \& Bourgeron, 2006). ASD-affects the social, behavioral, and intellectual development of individuals with different intensities. First symptoms start between the first and second year of life, which is confusing for the family, as a relatively normal developed child started to regress in their cognitive development, showing more and more disturbing behavior, which leads parents to seek medical help and finally to diagnosis (Ozonoff et al., 2009). For diagnosis, there are challenges, as alongside possible mental retardation, late speech skills, or not developing it at all, obsessive-compulsive behaviors (stereotypes), lack of interest in interaction with other people may lead to a different conclusion. ASD could be divided into high functioning, average, and deep autism. As for more severe, it is very hard to communicate due to the common lack of normal speech (alternative ways of communication as pictures are necessary). Thus it is difficult to determine the intellectual capacity of individuals, which often leads to the conclusion that they are also intellectually disabled. But their lack of interest in daily life activities, social interaction, and inability to communicate just give specialists no instruments to quantitative their performance in that manner, so also inability to reveal what is happening on a cognitive level leads to deep retardation conclusion (Lincoln et al., 1995)

Highly functioning ASD people could speak,-present logical reasoning; their motor development is close to normal. Mostly, they can interact with others but tend to avoid or ignore social activity. Social dynamics, flow, and coping with a group of people is hard for them due to sensory disturbances, as they can easily be overloaded with noise and information, which could lead to aggression or fleeing. Last but not least part of an often occurred trait in ASD generally, but also in high functioning ASD, is compulsive interest in a specific part of reality. All this is a crucial barrier toward attending regular school, as a crowded class and noise serve an un- bearable environment for such people, even if they are capable of learning served content. Presented traits and dependencies of development eventually lead to putting a child in the special education system (Wei et al., 2014).

Before that happens, often after diagnosis, ASD individuals started to attend rehabilitation sessions of any kind, such as physical, sensory, or speech therapy. But lack of social competencies and obsessive behaviors and interests leads them to avoid playing with other children, sports activities, as game rules are too complicated for them. While typically developed child tends to play a lot, explore the whole world and develops manually, the interests of ASD individuals are restricted by coordination and sensory disturbances. This lack of spontaneous physical activity from preschool life gave them worse motor competencies compared to their typically developed peers (Van Waelvelde et al., 2010). This inequality in development is tried to be compensated with different therapy, special physical education programs, but this stimuli load does not compensate for spontaneous physical activity. Some parts of motor development could be comparable to normally developed children, like cardiovascular capacity, but for high functioning individuals their main interest lies in cognitive stimuli linked to their mania (obsessive interest in the certain topic), so they will not spend their free time for any kind of aerobic activity (Paula, 2018).

Previously we mentioned that some of the learning problems could be linked to sensory disturbances. It is because some mental skills like abstract thinking, mathematics, and geometry are strictly connected with the ability to imagine placing in 3-Dimensional space and they do not possess that ability. They could perform simple arithmetic due to training, but things like geometry are beyond their cognition. This will also connect with tactics in sports games and imagination where the ball will be after interaction with other players (Orr et al., 2019).

Physical literacy covers far more than participation in physical education classes or training in sports sections (Culpepper \& Killion 2017). This term is interpreted as the motivation, confidence, physical competence, understanding, and knowledge that individuals develop for maintaining physical activity at an appropriate level throughout life. The main aim of the formation and development of physical literacy is to move with competence and confidence in a wide variety of physical activities in multiple environments that benefit the healthy development of the whole person (Whitehead, 2010; Duquette et al., 2016; Bukova et al., 2019). 
Physical literacy is interrelated with other subjects in school since the development of physically literate people is the primary purpose of school physical education. The aim of the formation of physical literacy during the education process is the empowerment of individuals to choose a physical activity for preserving a healthy life, which is known to enhance its quality (Ortenburger et al., 2017).

Quality of life is often defined as a subjective assessment of the positive and negative dimensions of one's functioning (Memisevic \& Djordjevic 2019). ASD affects nervous system development that undoubtedly has an impact on the quality of life. However, there is no clear understanding of the extent of this impact. Some studies show that children with physical disabilities experience a life quality similar to children with ASD. Others claim that children with ASD describe their quality of life as low. Also, according to some reports, the life quality of children with physical disabilities is similar to healthy children (Kuhlthau et al., 2010).

The level of physical literacy of children with ASD has not been studied; its relation to the quality of life is poorly investigated. This does not contribute to receiving important information about the general level of physical literacy and its separate elements, outlining the individual educational trajectories for improving a child's life quality.

There is a trend of placing ASD individuals with integration classes in schools or blending them to different disabilities in special facilities to enhance their development. But an inability to social interaction and the nature of common children, even when educated toward how they should behave toward such a person, may lead to exclusion from a group and isolation of ASD individuals (Chamberlain et al., 2007).

Lastly, all presented factors lead to the conclusion that the physical literacy of ASD individuals could be worse than normally developed equals. The degree of these changes is the main topic of this paper.

We seek to obtain knowledge of how ASD individuals differ from their normally developed equals in terms of physical literacy and quality of life in a stage of early adolescents. The obtained information will be possible because of highly functioning ASD individuals, who are able to express their introspective sensations into the survey, and alongside motor tests described in methods, we will have insight into differences between their development. The application aim will be to determine future goals of therapy to minimize the potential gap of motor competencies, and physical literacy of ASD individuals compare to normally developed individuals.

\section{MATERIAL AND METHODS}

\section{Participants}

Data have been collected from two samples: 18 males with autism spectrum disorders (ASD) aged 11-13 years (main group) and 30 males aged 11-12 years without ASD diagnosis (control group, CG). The participants in both groups were males. Test subjects were recruited between October 2018 and May 2019 at Lviv Centre for Rehabilitation and Training "Dovira" and schools in Lviv (Ukraine). Children with ASD were included in the main group if they were able to follow simple verbal instructions in Ukrainian. Children were excluded if they had a condition or injury that might affect their physical function. For samples height $(\mathrm{cm})$ and weight $(\mathrm{kg})$ were measured using a stadiometer and electronic scales, respectively. Measurements were taken three times, and mean values were calculated. Body mass index was calculated by dividing a participant's weight in kilograms by their height in meters squared (Table 1).

All data have been collected prospectively. Ethics approval has been obtained from the Ethics Committee of

Table 1. Profiles of Participants

\begin{tabular}{l|c|c|c|c}
\hline \multirow{2}{*}{ Profile } & \multicolumn{2}{|c|}{ Main group $(n=18)$} & \multicolumn{2}{c}{ Control group ( $n=30)$} \\
\cline { 2 - 5 } & $M \pm S D$ & $X_{\min }-X_{\max }$ & $M \pm S D$ & $X_{\min }-X_{\max }$ \\
\hline Age, years & $11.63 \pm 1.59$ & $11-13$ & $11.47 \pm 0.51$ & $11-12$ \\
\hline Body weight, $\mathrm{kg}$ & $44.53 \pm 10.58$ & $31-63$ & $42.97 \pm 7.86$ & $31-60$ \\
\hline Height, $\mathrm{cm}$ & $150.37 \pm 12.21$ & $132-171$ & $153.17 \pm 6.94$ & $140-165$ \\
\hline $\mathrm{BMl}, \mathrm{kg} / \mathrm{m}^{2}$ & $18.41 \pm 4.99$ & $14.7-24.9$ & $18.22 \pm 2.45$ & $14.2-23.3$ \\
\hline
\end{tabular}


Lviv State University of Physical Culture. Written informed consent for participating and publication of the results has been obtained from the parent or guardian of every child.

\section{Physical literacy}

Canadian Assessment of Physical Literacy - second edition (CAPL-2) (Longmuir et al., 2017)combined, and complex movement skills required to support childhood physical literacy. The secondary aim was to establish the feasibility, objectivity, and reliability evidence for the assessment. Methods An expert advisory group recommended a course format for the assessment that would require children to complete a series of dynamic movement skills. Criterion-referenced skill performance and completion time were the recommended forms of evaluation. Children, 8-12 years of age, self-reported their age and gender and then completed the study assessments while attending local schools or day camps. Face validity was previously established through a Delphi expert $(\mathrm{n}=19,21 \% \mathrm{fe}-$ male has been applied for assessing the level of physical literacy. This tool provides an assessment of 14 indicators, which in turn are combined into four domains of physical literacy: (1) daily behavior, (2) motivation and confidence, (3) physical competence, (4) knowledge and understanding. Domain valuation has been carried out in accordance with CAPL-2 recommendations or modifications.

Surveys have been used for evaluating the daily behavior domain, motivation and confidence domain, knowledge and understanding domain. Daily behavior has been accessed by a self-reported number of days per week with moderate/ vigorous physical activity. The questions concerned last week's activity (outdoor games, going in for sports, running, dancing, swimming, and even playing snowballs). The valuation has been done on the following parameters: less than four days a week - beginning; 4 days a week - progressing; 5-6 days a week - achieving; more than six days a week - excelling.

Motivation and confidence have been evaluated by six questions. Question scores ranged from 0.5 to 2.5 points (intrinsic motivation subdomain), or $0.6-2.5$ points (competence subdomain). The following rating scale has been used for rating this domain: less than 16.8 points - beginning; 16.8-23.7 points - progressing; 23.8-26.2 points - achieving; more than 26.2 points - excelling.

Knowledge and understanding assessment form consist of 5 questions, the maximum number of points for the first four -4 points, for the fifth question -6 .
The following summary scale for this domain has been used: less than 5.6 points - beginning; 5.6-7.8 points progressing; 7.9-8.6 points - achieving; more than 8.6 points - excelling.

While performing some evaluation tests for physical competence, children with ASD have faced a number of challenges. Thus, daily motor activity has been determined solely on the basis of the self-report of children (and their guardians); the beep test and the Canadian agility and movement skill assessment (CAMSA exercise) have not been performed due to the performing difficulties with ASD children; instead of a beep test, all participants performed a standard load test of 30 squats in 45 seconds, etc. In general, the tests used at the Al-Shaffal Rehabilitation Center (Doha, Qatar) have been used to assess the level of physical competence. Most of the test exercises in it are similar to the structural elements of an integrated exercise CAMSA. The evaluation of the quality of motor skills has been carried out according to a 4-point system developed at the Al-Shaffall Rehabilitation Center $(3$ - the participant performed the exercise without errors, 2 - with some minor errors, 1 - with blunders, 0 - failed to meet the requirements). When working with children with ASD, oral explanations and/ or pictures and/ or presentations have been used as needed to help the children understand the task.

Since, according to the recommendations of the CAPL-2 methodology, analysis for each domain (or subdomain) is acceptable, the final assessment of the level of physical literacy has not been figured out (CAPL Manual, n.d.). All modifications of the CAPL-2 methodology are shown in Table 2.

The level of functional and reserve capabilities of the cardiovascular system of children was determined by the duration of the heart rate recovery time (to the initial level) after a standard load of 30 squats for 45 seconds according to the following formula:

$$
\text { Index }=\left(4 \times\left(H \boldsymbol{R}_{1}+H R_{2}+H R_{3}\right)-200\right) \times \mathbf{1 0}^{-1}
$$

After 5 minutes of rest, the study participant had a heart rate measured for $15 \mathrm{sec}$ in a sitting position $\left(\mathrm{HR}_{1}\right)$, then he performed 30 deep squats in $45 \mathrm{sec}$, specifically, when squatting, his hands were raised up, and when getting up, they were lowered down, the participant was counting squats performed independently aloud (in order to maintain proper breathing). Afterward, the participant immediately sat on a chair. Heart rate was calculated for the first $15 \mathrm{sec}$ of the first minute of rest $\left(\mathrm{HR}_{2}\right)$, then - for the first $15 \mathrm{sec}$ of second minutes of rest $\left(\mathrm{HR}_{3}\right)$. 
Table 2. Features of physical literacy domain assessment using the CAPL-2 methodology (with adjustments used in the study)

CAPL-2 indicators, unit of measurement

Compliance with the Protocol, test exercises used in the study

\section{Daily behavior domain, max 30 points}

1.1. Self-reported number days a week a child engages in MVPE (moderate-to-vigorous-PA), number days a week

1.2. Average daily step count (pedometer), max 25 points NA

\section{Motivation and confidence domain, max 30 points}

2.1. Motivation and competence (questionnaire $-2 \times 3$ items, max 15 points)

2.1.1. Intrinsic motivation ( 3 items, $\max 7.5$ points)

2.1.2. Competence (3 items, $\max 7.5$ points)

2.2. Predilection and Adequacy (questionnaire $-2 \times 3$ items, max 15 points)

2.2.1. Predilection ( 3 items, $\max 7.5$ points)

2.2.2. Adequacy (3 items, max 7.5 points)

\section{Physical competence domain, max 30 points}

Progressive aerobic cardiovascular endurance run (PACER) shuttle run, max 10 points

\begin{tabular}{|c|c|}
\hline & \\
\hline Plank, max 10 points & + \\
\hline CAMSA (assessment of time and skill, max 10 points) & * \\
\hline - Jumping on 2 legs from circle to circle & + \\
\hline - Sliding & NA \\
\hline $\begin{array}{l}\text { - Catching the ball after passing by the teacher } \\
\text { - Throwing the ball into the target }\end{array}$ & $\begin{array}{l}\text { Passing and catching the ball } \\
\text { Pushing a medicine ball }\end{array}$ \\
\hline - Hopping in motion & NA \\
\hline - Single-leg jumps & Long jump* \\
\hline - Kick at the goal & Test "4 balls"” \\
\hline- & $20 \mathrm{~m} \mathrm{run}^{* *}$ \\
\hline- & $4.5 \mathrm{~m}$ tight-rope walking ${ }^{* *}$ \\
\hline- & Keeping the "Flamingo" pose on bench" \\
\hline- & Clapping hands* \\
\hline- & Writing letter "O"** \\
\hline & Two-leg squats ${ }^{* *}$ \\
\hline
\end{tabular}

\section{Knowledge and understanding domain, max 10 points}

Knowledge and understanding (questionnaire, max 10 points)

$+$

Notes. + - Applied in original form; NA - not applied; *Applied in adapted form; ** Used in addition to CAPL-2.

The estimated test result has been carried out in accordance with the modified assessment method developed by Zanevsky (Zanevsky \& Zanevska, 2013) for children and adolescents (Table 3).

Jumping on 2 legs from circle to circle. Six hoops of 0.63 $\mathrm{m}$ in diameter have been laid down on the floor in two rows of 3 hoops in each, they have been fastened with adhesive tape to each other and stuck to the floor. The participants stood in front of the starting line. Their task was to perform six wave jumps on one leg (Fig. 1) from one hoop to another. A stopwatch was switched on upon the command "Go!" and switched off when the participant jumped 
Table 3. Evaluation of functional reserve levels of the cardiovascular system for secondary school-age students

\begin{tabular}{|c|c|c|c|c|c|c|}
\hline \multirow{3}{*}{ Level } & \multicolumn{6}{|c|}{ Age, years } \\
\hline & 10 & 11 & 12 & 13 & 14 & 15 \\
\hline & \multicolumn{6}{|c|}{ Test results } \\
\hline $1-$ low & $\geq 21.4$ & $\geq 19.4$ & $\geq 18.2$ & $\geq 17.0$ & $\geq 15.7$ & $\geq 15.1$ \\
\hline 2 - below average & $15.4-21.3$ & $13.7-19.3$ & $12.7-18.1$ & $11.6-16.9$ & $10.6-15.6$ & $10.0-15.0$ \\
\hline 3 - average & $11.8-15.3$ & $10.3-13.6$ & $9.3-12.6$ & $8.4-11.5$ & $7.4-10.5$ & 7.0-9.9 \\
\hline 4 - above average & $8.2-11.7$ & $6.9-10.2$ & $6.0-9.2$ & $5.2-8.3$ & $4.3-7.3$ & $3.9-6.9$ \\
\hline 5 - high & $\leq 8.1$ & $\leq 6.8$ & $\leq 5.9$ & $\leq 5.1$ & $\leq 4.2$ & $\leq 3.8$ \\
\hline
\end{tabular}

into the 6th hoop. Each participant has had two attempts; the time of the best one has been recorded into the protocol. The total time required to complete the test, and the quality of the technique have been evaluated.

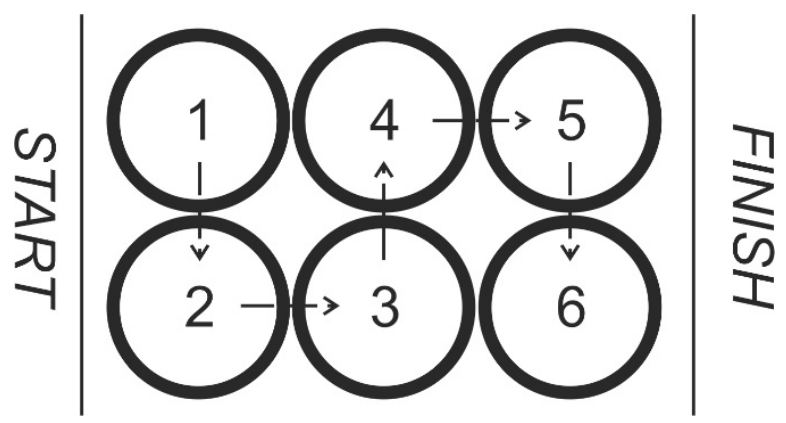

Fig. 1. Scheme of exercises with jumping directions.

The plank test has been performed to measure the strength and endurance of muscles, press, and back, as well as chest muscles, shoulders, anterior thigh, and buttocks. The participant lies down on the floor with his abdomen down, bends his elbows at right angles, and rests only on the forearms and toes. That body position has to be maintained for as long as possible. The test is to be completed when the participant cannot hold his back straight and his hips are lowered. The time (in seconds) and technique mastery have been recorded.

Passing and catching the ball with two hands has been used to assess agility. At a distance of $2 \mathrm{~m}$ far away from the wall, a line has been drawn on the floor which is not to be approached closer to the wall; a target of $1 \times 1 \mathrm{~m}$ in size has been drawn on the wall, centered at the participant's eye level ( $1 \mathrm{~m}$ from the floor up to the lower edge of the target). During the exercise, it has not been allowed to hit the ball with hands - but only catch and throw (as in basketball). The participants have made two attempts; the number of goals caught in $30 \mathrm{sec}$ has been recorded into the protocol at the best attempt.
Pushing a medicine ball (1 kg of weight) has been performed with two hands from the chest in a sitting position, leaning back against the wall. The test has been conducted to determine the speed of arms and the ability to pass the ball with two hands from the chest. The distance from the wall to the place where the ball landed has been recorded. The best of 2 attempts has been registered, and the exercise technique has been evaluated.

The long jump from the spot has been used to determine the level of development of the high-speed force; the test participant has to stay with his toes directed to the line, swing his arms back, then sharply move them forward, and jump as far as possible pushing away with two legs. The result of the test is the jump range $(\mathrm{cm})$, taking into account the results of the best of the two attempts.

The test exercise " 4 balls" has been carried out to determine the agility and mastery degree of passing (kicking) a soccer ball. For the test (Fig. 2), four soccer balls have been placed in the center of a $6 \times 6 \mathrm{~m}$ court in a circle with a diameter of $2 \mathrm{~m}$, each at a distance of $2 \mathrm{~m}$ to the goal marked with cones; a mark has been drawn in the center

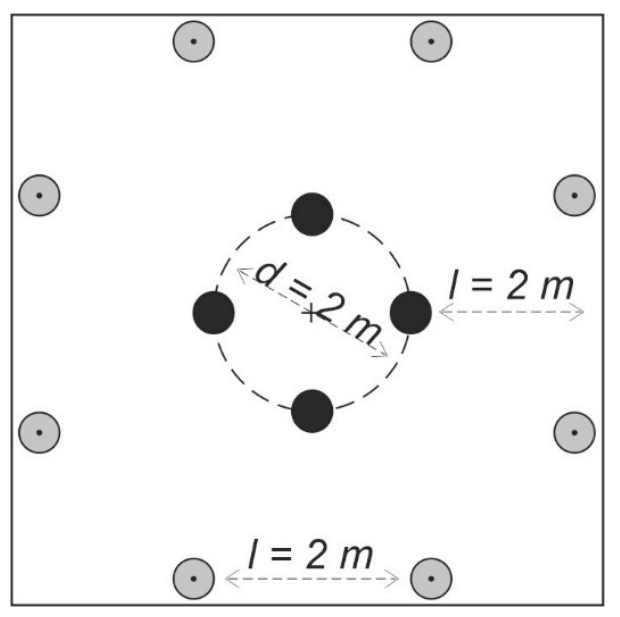

Fig. 2. Layout of items for the test "4 balls" 
of the circle. The participant had to stand in the center of the circle, on command he approached one ball, kicked it into one "goal" and returned to the center of the circle, then tried to kick another ball into the other "goal," etc. The stopwatch was stopped after four kicks and returned to the center of the circle. The best result of two attempts has been recorded in the protocol. The exercise execution time (in seconds) has been evaluated, and the technique for transferring the ball has been assessed on a 4-point scale.

$4.5 \mathrm{~m}$ tight-rope walking. This task is adapted from the tandem walking test (Sigmundsson et al., 2016) the psychometric properties of a new test battery aimed at quantifying motor competence across the life span are explored. The battery was designed to be quantitative, simple to administer, applicable for large-group testing, and reliably to monitor life span motor development. A total of 638 participants between 5 and 83 years of age completed assessment of four different motor tasks (two fine and two gross motor tasks (Rooks et al., 1997) most have used exercises performed at specific intensities on expensive equipment, which limit their widespread applicability. We tested whether two self-paced, less expensive exercise protocols could be effective and safe for modifying neuromotor performance and functional capacity in community-dwelling adults 65-95 years of age. Methods. One hundred and thirty-one subjects were randomized to a novel resistance training, walking, or control group. Subjects determined their level of resistance or walking intensity self-paced (Rinne et al., 2001) and is considered to be a measure of dynamic balance capabilities. Participants are required to walk down a straight line $(4.5 \mathrm{~m}$ long) marked on the floor as fast as they can place their heel against the toes of the foot in each step.

Scales for evaluation of the child's psychophysical preparedness (clapping hands, writing the letter "O," number of two-leg squats in 10 seconds, keeping the "Flamingo" pose) have been described in (Pavlova et al., 2018).

\section{Health-related quality of life (HRQOL)}

Pediatric Quality of Life Inventory (PedsQL ${ }^{\mathrm{rm}} 4.0$ ) has been used to assess the quality of life (Varni et al., 2007; Varni et al., 1999). In order to apply the Generic Core and Cognitive Functioning Scales, a User Agreement has been signed with the Mapi Research Trust (Lyon, France), and special permission has been received to conduct the survey. The Ukrainian version of the questionnaire has been confirmed.

Generic Core consists of separate units; the total number of questions is 23 . A child gives answers to questions about problems with health and performing dif- ferent activities for the last month. A five-point Likert scale from 0 (never) to 4 (almost always) has been used. The results of the survey have been calculated according to the four scales - Physical Functioning (8 questions), Emotional Functioning ( 5 issues), Social Functioning (5 questions), and School Functioning (5 issues). Also, the Psychosocial Health Summary score (sum of the items over the number of items answered in the Emotional, Social, and School Functioning scales) and Physical Health Summary Score (the same as Physical Functioning Scale score) have been calculated.

The quality of life has been accessed according to the 100 -point scale. Higher scores indicate better HRQOL Indicators in the range of $0-50$ points have been interpreted as poor quality of life, $50-75$ points - average, and 75-100 points - high.

\section{Statistical analysis}

Data have been analyzed descriptively using average (M), the corrected mean square deviation (SD), the smallest value $\left(\mathrm{X}_{\min }\right)$, the highest value $\left(\mathrm{X}_{\max }\right)$, associated $95 \%$ confidence intervals were also calculated (95\% CI).

Differences between groups have been determined using the Kruskal-Wallis ANOVA, the probability was set at $\mathrm{p}<0.05$.

The close linkage has been established by the Brave-Pearson correlation coefficient.

\section{RESULTS}

\section{Physical Literacy}

The motivation and confidence domain in children with ASD made up $18.66 \pm 3.31$ points and was statistically significantly lower $(\mathrm{p}<0.05)$ compared with the control group (25,32 $\pm 3,39$ points) (Table 4$)$.

The knowledge and understanding domain of children in the main and control groups was below the average level and was not statistically significantly different.

Physical activity volumes of children with ASD did not differ from those of healthy children: the number of days during the week when healthy children and children with ASD were physically active for more than $60 \mathrm{~min}$ per day, are similar ( $4.30 \pm 1.88$ for the control group; $3.13 \pm 1.31$ days for the main group).

The results of all test exercises (Table 5) of the children from the main group were significantly lower than those from the control group. Statistically credible differences were observed in the performance of all tests, in particular, the results of performing the jumping on 2 legs from circle to circle of the main group participants 
Table 4. The level of physical literacy

\begin{tabular}{|c|c|c|}
\hline Domains and subdomains & Main group, $M \pm S D$ & Control group, $\mathrm{M} \pm \mathrm{SD}$ \\
\hline \multicolumn{3}{|l|}{ 1. Daily behavior domain } \\
\hline $\begin{array}{l}\text { 1.1. Self-reported number days a week a child engages in MVPE, } \\
\text { days/ week }\end{array}$ & $3.13 \pm 1.31$ & $4.30 \pm 1.88$ \\
\hline Level & Beginning & Progressing \\
\hline
\end{tabular}

2. Motivation and confidence domain

\begin{tabular}{|c|c|c|c|}
\hline \multirow{2}{*}{ 2.1. Motivation and competence, points } & Motivation & $5.97 \pm 1.94$ & $7.05 \pm 0.56$ \\
\hline & Competence & $3.75 \pm 1.46$ & $5.50 \pm 1.59$ \\
\hline \multirow{2}{*}{ 2.2. Predilection and Adequacy, points } & Predilection & $4.61 \pm 1.09$ & $6.62 \pm 1.04$ \\
\hline & Adequacy & $4.34 \pm 1.31$ & $6.14 \pm 0.96$ \\
\hline \multicolumn{2}{|l|}{ Overall number of points } & $18.66 \pm 3.31^{*}$ & $25.32 \pm 3.39$ \\
\hline \multicolumn{2}{|l|}{ Level } & Progressing & Achieving \\
\hline \multicolumn{4}{|l|}{ 4. Knowledge and understanding domain } \\
\hline \multicolumn{2}{|l|}{ Overall number of points } & $5.06 \pm 1.88$ & $3.67 \pm 1.92$ \\
\hline \multicolumn{2}{|l|}{ Level } & Beginning & Beginning \\
\hline
\end{tabular}

Notes. *Significant differences at $p<0.05$

have been 3 times lower, keeping the "Flamingo" pose on a bench -2 times lower, plank exercise -6 times lower, writing the letter "O" -2.5 times lower compared with participants in the control group.

ASD children's static force endurance of legs and trunk muscles (84\%) is left far behind the norm. The results of test exercises that require high accuracy of muscle force, the direction of movements and their rapid change, coordination (consistency) of work of muscles of arms, legs and trunk, dynamic and static balance, etc. (by 67-45\%) are left significantly behind the norm. In wellknown exercises and workouts with a relatively simple movement structure (running $20 \mathrm{~m}$, clapping, squatting on two legs, long jumping, etc.), children with ASD lag behind less (by 30-40\%).

\section{Health-related quality of life}

The quality of life of children with ASD is below average (Physical Functioning - 59.54 points) and of low level (Psychosocial Health Summary - 48.77 points) (Table 5). Confidence intervals for the main group have been within the narrow range $(95 \% \mathrm{Cl}$ for the scale of Physical Functioning - 51.16-67.92 points; for the scale of psychosocial status - 43.73-53.82 points). Statistically credible differences between the results of the main and control groups were observed on the scales of Physical and
Social Functioning, as well as School Functioning. The quality of life on Social Functioning appeared to have the lowest rates among the children of the main group. In the control group, most indicators have been in the high range, with an average quality of life (69.50 points) observed on the Emotional Functioning scale. The quality of life on the Social Functioning scale was critically low for the children of the main group; this indicator has been two times lower than that one of the control group.

A detailed analysis of the respondent's answers showed some differences in the Physical Functioning of persons of main and control groups (Table 6). A large number of children of the main group reported varying degrees of difficulties while running (47.36\%), lifting heavy objects $(68.42 \%)$, taking part in physical education, and going in for sports (47.37\%). The following usually does not create any discomfort for children of this group: walking $(73.69 \%)$, self-care (taking a shower or having a bath independently $-63.16 \%$ ); half of the respondents have had no complaints on pain (47.37\%) or low energy level $(47.37 \%)$. The vast majority of control group respondents (more than $70 \%$ ) have never or almost never had problems in their daily activities.

The level of Emotional Functioning of the main group (58.42 \pm 19.51 points) did not differ from that one of the control group. Almost the same number of 
Table 5. Physical literacy Indicators for children with ASD and healthy peers

\begin{tabular}{|c|c|c|c|c|c|}
\hline \multirow{2}{*}{ Test } & \multicolumn{2}{|c|}{ Main group } & \multicolumn{2}{|c|}{ Control group } & \multirow{2}{*}{$\begin{array}{c}\text { Difference (in \%) } \\
\text { between } \\
\text { MG and CG }\end{array}$} \\
\hline & M & SD & M & SD & \\
\hline $\begin{array}{l}\text { Level of functional and reserve capacity } \\
\text { of the cardiovascular system }\end{array}$ & 12.04 & 1.82 & 14.44 & 3.83 & \\
\hline Jumping on 2 legs from circle to circle, sec & $11.89^{*}$ & 3.23 & 3.91 & 1.08 & 67.1 \\
\hline Passing and catching the ball, amount in $30 \mathrm{sec}$ & $11.62^{*}$ & 6.42 & 21.6 & 7.36 & 46.2 \\
\hline Pushing a medicine ball, $\mathrm{cm}$ & $276.14^{*}$ & 101.17 & 720.17 & 244.53 & 61.7 \\
\hline Long jump, cm & $94.18^{*}$ & 33.2 & 158.7 & 16.94 & 40.7 \\
\hline Test "4 balls", sec & $9.18^{*}$ & 2.7 & 5.09 & 2.04 & 44.6 \\
\hline $20 \mathrm{~m}$ run, sec & $5.71^{*}$ & 0.89 & 4.01 & 0.52 & 29.8 \\
\hline $4.5 \mathrm{~m}$ tight-rope walking, sec & $15.03^{*}$ & 6.27 & 6.95 & 1.19 & 53.8 \\
\hline Keeping the "Flamingo" pose on the bench, sec & $30.49^{*}$ & 24.53 & 59.95 & 34.01 & 49.1 \\
\hline Plank test, sec & $22.35^{*}$ & 32.82 & 136.6 & 103.28 & 83.6 \\
\hline Clapping hands, amount in $10 \mathrm{sec}$ & $27.79^{*}$ & 6.81 & 40.6 & 14.58 & 31.6 \\
\hline Writing the letter "O", amount in $10 \mathrm{sec}$ & $9.43^{*}$ & 3.52 & 23.57 & 4.77 & 60.0 \\
\hline Two-leg squats, amount in $10 \mathrm{sec}$ & $7.91^{*}$ & 1.64 & 12.73 & 2.52 & 37.9 \\
\hline
\end{tabular}

Note. *Differences are credible when $p<0.05$

children complained about feeling of anger (16.67\% of the control group, $15.79 \%$ of the main group), only sometimes were angry $46.67 \%$ of the control group and $52.63 \%$ of main group, almost never sad $-43.33 \%$ of control group and $36.84 \%$ of the main group, never worry about what would happen $-36.67 \%$ control group, $26.32 \%$ main group. Most children $(70.0 \%$ control group, $63.16 \%$ main group) indicated that they rarely have problems with falling asleep. The relative number of persons suffering from sleep disorders was $15.79 \%$ of the main group and $10.0 \%$ of the control group. Also, in the main group, every fifth $(21.05 \%)$ child often or almost always felt fear/ was afraid of something, whereas, in the control group, there were no such complaints.

The low indicator on the Social Functioning scale ( $40.26 \pm 13.89$ points) of the main group is due to difficulties in establishing good relations with other children (47.37\%), maintaining friendly relations (36.84\%), and experience of being bullied (21.05\%). Among the complaints of children, there were the following: inability to do the same their peers do (68.42\%), and inability to keep up with the pace of play of other children (68.43\%). Respondents of the control group (96.67-93.34\%) stat- ed that they did not have any problems regarding various aspects of social communication.

The low quality of life rate on the School Functioning (47.63 \pm 9.77 points), and Cognitive Functioning (39.54 \pm 14.56 points) of the main group, have been caused by the presence of a large number of people with problems of concentration during the lesson (47.37\%), memorizing educational material $(52.64 \%)$, lacking ability to keep up with their studies (31.58\%). A significant proportion of ASD children cannot think quickly (68.42\%), 63.16\% experienced difficulties in keeping a focus on the task, $42.1 \%$ had difficulties with remembering what they have been already told, $47.37 \%$ had difficulties with remembering what they have just heard, $47.37 \%$ had a hard time remembering what they have just thought of.

\section{Correlation analysis}

There were numerous positive and negative correlations between quality of life and some indicators of physical literacy among children with ASD (Table 6). A close relationship was found between the results of different life quality parameters and indicators of physical literacy: 
Table 6. Quality of life for children of the main and control groups

\begin{tabular}{|c|c|c|c|}
\hline \multirow{2}{*}{ Scales } & \multicolumn{3}{|c|}{ Main group } \\
\hline & $\mathrm{M} \pm \mathrm{SD}$ & $95 \% \mathrm{Cl}$ & $X_{\min }-X_{\max }$ \\
\hline Physical Functioning & $59.54 \pm 17.38^{*}$ & $51.16 ; 67.92$ & $25.00-90.63$ \\
\hline Psychosocial Health Summary & $48.77 \pm 10.47^{*}$ & $43.73 ; 53.82$ & $26.67-71.67$ \\
\hline Emotional Functioning & $58.42 \pm 19.51$ & $49.02 ; 67.83$ & $0.00-80.00$ \\
\hline Social Functioning & $40.26 \pm 13.89^{*}$ & $33.57 ; 46.96$ & 20.00-65.00 \\
\hline School Functioning & $47.63 \pm 9.77^{*}$ & $42.92 ; 52.34$ & $25.00-70.00$ \\
\hline \multirow[t]{2}{*}{ Cognitive Functioning } & $39.54 \pm 14.56$ & $32.52 ; 46.56$ & $16.67-66.67$ \\
\hline & \multicolumn{3}{|c|}{ Control group } \\
\hline Physical Functioning & $88.13 \pm 10.20$ & $84.32 ; 91.93$ & $62.50-100.00$ \\
\hline Psychosocial Health Summary & $78.06 \pm 9.26$ & $74.60 ; 81.52$ & 61.67-95.00 \\
\hline Emotional Functioning & $69.50 \pm 17.04$ & $63.14 ; 75.86$ & $25.00-100.00$ \\
\hline Social Functioning & $91.50 \pm 8.42$ & $88.35 ; 94.65$ & $70.00-100.00$ \\
\hline School Functioning & $73.17 \pm 13.99$ & $67.94 ; 78.39$ & $50.00-100.00$ \\
\hline Cognitive Functioning & \multicolumn{3}{|c|}{ ND } \\
\hline
\end{tabular}

Notes. "Statistically credible differences $(p<0.05)$ between the main and control groups; ND - not determined

jumping on 2 legs from circle to circle and Physical Functioning ( $\mathrm{r}=-0.699)$, Emotional functioning $(\mathrm{r}=-0.547)$, Social Functioning $(\mathrm{r}=-0.820)$, School Functioning $(\mathrm{r}=-0.622)$, Cognitive Functioning $(\mathrm{r}=-0.509)$; the results of the long jump test and Emotional Functioning $(\mathrm{r}=0.724)$; the results $20 \mathrm{~m}$ run and Emotional Functioning $(\mathrm{r}=-0.586)$, and School Functioning $(\mathrm{r}=-0.548)$.

Cognitive functioning was associated with knowledge and understanding parameters $(\mathrm{r}=0.632, \mathrm{p}<0.001)$.

\section{DISCUSSION}

The low subjective evaluation of own physical competence in children with ASD was confirmed by tests of physical fitness. Results of all testing exercises had significantly lower values compared to that of children of the same age group. Our study confirms the results obtained by researchers (Law et al., 2018; MacDonald, Esposito \& Ulrich, 2011; Pan et al., 2016; Pace \& Bricout, 2015; Pan, 2014; Bricout et al., 2018; Jansiewicz et al., 2006; Downey \& Rapport, 2012) on a significant lag in the development of physical qualities in children with ASD. In accordance with the results of the conducted study, significant differences were observed in indicators of strength and endurance. Children with ASD have the most signif- icant lag in static strength endurance of muscles in arms, legs, body (84\%), results of test exercises that require high accuracy of muscular efforts, directions of movements and their quick change, coordination (coherence) of working muscles in arms, legs, body, dynamic and static balance lag behind the standard (by $67 \%$ to $45 \%$ ).

When performing simple exercises and exercises with a relatively simple structure of movements (running for a distance of 20 meters, handclap, two-leg squat, long jump), children with ASD showed a lag less (by 30\% to 40\%). This indicates that in addition to static strength endurance, children with ASD have significantly affected coordination.

Researchers (Manjiviona \& Prior, 1995; Dewey et al., 2007; Staples \& Reid, 2010) claim that autistic spectrum disorders are often accompanied by disturbances of motor skills. The majority (59\%) of children with ASD have a satisfactory level of physical fitness (Dewey et al., 2007); they perform movements at motor level inherent to children who are twice younger (Staples \& Reid, 2010). It should be noted that some research workers (Tyler et al., 2014) did not observe differences between the results of healthy children and children with ASD based on such tests as the beep test, trunk bending, but found a significant difference $(\mathrm{p}=0.03)$ in hand strength. 
Table 7. Correlation between indicators of life quality and physical literacy of children with ASD

\begin{tabular}{|c|c|c|c|c|c|c|}
\hline \multirow[b]{2}{*}{ Domains and subdomains of physical literacy } & \multicolumn{6}{|c|}{ Quality of Life Scales } \\
\hline & 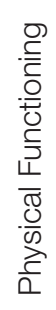 & 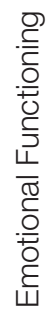 & 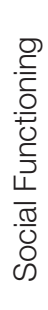 & 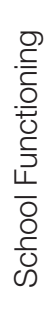 & 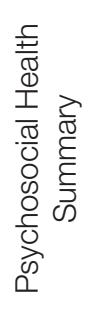 & 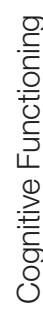 \\
\hline
\end{tabular}

\section{Daily behavior domain}

\begin{tabular}{|c|c|c|c|c|c|c|}
\hline $\begin{array}{l}\text { Self-reported number days a week } \\
\text { a child engages in MVPE }\end{array}$ & -0.013 & 0.029 & -0.153 & -0.137 & -0.531 & 0.049 \\
\hline \multicolumn{7}{|l|}{ 2. Motivation and confidence } \\
\hline Motivation and confidence & 0.072 & -0.170 & -0.340 & -0.152 & -0.489 & -0.108 \\
\hline \multicolumn{7}{|l|}{ 3. Physical competence } \\
\hline Passing and catching the ball & -0.076 & 0.438 & -0.398 & 0.170 & -0.233 & 0.184 \\
\hline Plank & -0.560 & 0.254 & -0.413 & -0.010 & -0.503 & 0.349 \\
\hline Keeping the "Flamingo" pose on the bench & -0.564 & 0.231 & -0.226 & 0.009 & -0.456 & 0.337 \\
\hline $4.5 \mathrm{~m}$ tight-rope walking & 0.579 & -0.244 & -0.153 & -0.278 & -0.163 & -0.431 \\
\hline 20 m run & -0.001 & -0.586 & -0.363 & -0.548 & 0.077 & -0.240 \\
\hline Jumping on 2 legs from circle to circle & -0.699 & -0.547 & 0.820 & 0.622 & 0.214 & 0.509 \\
\hline Long jump & 0.449 & 0.724 & -0.096 & 0.161 & 0.212 & -0.240 \\
\hline Test "4 balls" & -0.182 & 0.004 & 0.565 & 0.357 & 0.319 & -0.307 \\
\hline Pushing a medicine ball & -0.227 & 0.370 & 0.006 & 0.153 & -0.184 & 0.179 \\
\hline \multicolumn{7}{|l|}{ 4. Knowledge and understanding } \\
\hline Knowledge and understanding & 0.027 & -0.256 & -0.183 & -0.169 & -0.411 & 0.632 \\
\hline
\end{tabular}

The level of motor activity of all children examined was low, healthy respondents had 1-hour vigorous physical activity of $4.30 \pm 1.88$ days per week, while children with ASD had $3.13 \pm 1.31$ days per week. The findings differ significantly from the recommended value of 6 to 7 days per week. Similar results were obtained in studies by other researchers (Bricout et al., 2018), but they differ from the results of accelerometry and pedometry (Tyler et al., 2014). According to these data, the duration of light, moderate, and vigorous physical activity in children with ASD and healthy children of the same age were significantly different. In the first place, such differences between our data and the data of other researchers can be explained by the method used for meas- uring physical activity and the child's inability to assess the intensity of their physical activity. Although the obtained subjective data are not informative because they were not compared with objective indicators, the selected study method can be considered sufficiently sensitive.

Children with ASD are highly motivated to participate in physical activity; they understand the benefits of physical exercise, want to be stronger, quicker, and fitter. However, the success of these children in physical activity is low (3.75 \pm 1.46 points out of possible 7.5 points). They do not have skills in many sports and realize that they are inferior to other peers in physical fitness. The level of success is the lowest compared to other indicators of physical literacy. However, it should be noted that 
the level of competence of children in the control group $(5.50 \pm 1.59$ points) is the lowest compared to other components of the motivation and confidence domain.

Taking into account that the level of efficiency of the cardiovascular system of children with ASD (12.04 \pm 1.82 points) did not differ $(\mathrm{p}>0.05)$ from that of healthy children (14.44 \pm 3.83 points), we believe that the cause of the worst indicators of physical fitness is a lower level of physical activity of children with ASD. Thus, it is very encouraging that children with ASD significantly improve their physical qualities through specially designed programs of physical training in swimming, horse riding, martial arts.

The level of theoretical training of children with ASD is higher compared to that of their healthy peers. Children with ASD obtained better scores for the knowledge and understanding domain $(5.06 \pm 1.88$ points), but the level of knowledge of children in both the main and control groups was low, it was evaluated as the beginner level. This demonstrates the need for strengthening measures aimed at promoting physical education and sport among schoolchildren.

To evaluate the quality of life of children with ASD, the presence of the control group was crucial. Methods of evaluation usually take into account the cultural differences of inhabitants of different countries, but the quality of life of even healthy respondents may differ significantly. Thus, the constant decline in describing emotional functioning is inherent to Ukrainian respondents of all ages.

According to the Physical Functioning scale, the quality of life in children with ASD was close to the low level. The most common is the difficulty in daily activities, but most of the children did not complain of low energy levels or problems during the performance of physical exercises. The results of healthy respondents involved in the study indicate that most of them do not have any problems with moving, coping with stairs, and carrying loads.

The quality of life in accordance with the Emotional Functioning scale is relatively low both in children with ASD (58.42 \pm 19.51 points) and healthy respondents (69.50 \pm 17.04 points). However, in children with ASD, it is one of two indicators that have the highest value, while, in healthy respondents, it is one of the indicators that is significantly lower than the others.

According to the obtained data, $15.79 \%$ of children with ASD had constant complaints of sleep disturbance. Among healthy children, this indicator was 3.3\%. In various studies, it is indicated (Devnani \& Hegde, 2019; Krakowiak et al., 2008; Taira, Takase \& Sasaki 1998; Liu et al., 2006) that children and adolescents with autism suffer more often ( $40 \%$ to $80 \%$ ) from sleep problems, particularly insomnia. This problem is typical even for very young patients; $53 \%$ of persons aged 2 to 5 years had trouble sleeping (Krakowiak et al., 2008). Among the most common complaints, there are difficulties in falling asleep, insomnia, frequent awakening, early awakening, insomnia.

ASD is characterized by persistent violations of social interaction and communication. In the control group, $90 \%$ of respondents rarely have any difficulties during the interaction with other children, while respondents with ASD indicate that it is hard for them to establish good relationships with other children (47.37\%), had complaints about bullying $(21.05 \%)$ and noted that they could not do what other children can do $(68.42 \%)$, to maintain the required pace of the game (68.43\%).

Those responses from the ASD group gave information not only about themselves but also about the condition of the social environment and level of understanding about people with different disabilities by health peers. Establishing relationships could be easier with a better approach by health peers, especially with a high rate of bullying, probably to the different behavior of children with ASD (Duquette et al., 2016).

Our findings show, that there is a field for lots of improvements in terms of physical literacy for children in Ukraine, not only for those with ASD. The possible implementation value of obtained knowledge could form recommendations and application for better adjustment of therapy in special facilities for children of special needs, but also enhancement of integration on a field of sport between healthy and disabled children. More educational work by teachers is needed to increase tolerance for the disabled and also physical activities of children with ASD should be more directed into participating in the games with others (Chovanova, 2019), as this study reveals that they are longing for feeling like their healthy peers.

\section{CONCLUSIONS}

Children with ASD have a lower level of some indices of physical literacy (general motivation and confidence domain, physical competence indicators), and quality of life (according to scales of physical, social, and school functioning) compering with healthy peers. At the same time, however, the volume of physical activity, especially the vigorous, was very low and the same for the participants of the main and control group. The knowledge and understanding domain, no matter of group, was assessed 
at the beginning level. Physical literacy indicators of children with ASD are connected with the health-related quality of life of children with ASD. Lower values of indicators of a physical literacy test for ASD children are connected with their ability to participate in playful time with their peers. Due to obtained results and sufficient level of functional and reserve capacity of the cardiovascular system, physical education programs should focus more on coping with disabled children in the integration environment and better preparation of ASD children for physical activity, which requires social interaction like playing sports games.

\section{ACKNOWLEDGMENTS}

None.

\section{DISCLOSURE STATEMENT}

No potential conflict of interest was reported by the authors.

\section{FUNDING}

None.

\section{REFERENCES}

Bricout, V.A., Pace, M., Dumortier, L., Baillieul, F., Favre-Juvin, A., \& Guinot, M. (2018). Reduced Cardiorespiratory Capacity in Children with Autism Spectrum Disorders. Journal of Clinical Medicine, 7(10), 361.

Bukova, A., Hagovska, M., Drackova, D., Horbacz, A., Wasik, J., Krucanica, L. (2019). Awareness of patients suffering from selected chronic diseases of the importance of physical activity in treating their disorders. Physical Activity Review, (7), 234-239.

Pan, C.Y., Tsai, C.L., Chu, C.H, Sung, M.C., Ma, W.Y., Huang C.Y. (2016). Objectively measured physical activity and health-related physical fitness in secondary school-aged male students with autism spectrum disorders. Physical Therapy, 94(4), 511-520.

Chamberlain, B., Kasari, C., \& Rotheram-Fuller, E. (2007). Involvement or isolation? The social networks of children with autism in regular classrooms. Journal of Autism and Developmental Disorders, 37(2), 230-242.

Chovanova, E. (2019). The development of coordination abilities in non-integrated students and correction of behavior disorders in integrated students through engaging in movement games. Physical Activity Review, 7, 209-218.

Culpepper, D., Killion, L. (2017). Effects of Exercise on Risk-Taking, Physical Activity Review. 5, 1-5.

Devnani, P. A., \& Hegde, A. U. (2015). Autism and Sleep Disorders. Journal of Pediatric Neurosciences, 10(4),304-307.

Dewey, D., Cantell, M., \& Crawford, S. G. (2007). Motor and gestural performance in children with autism spectrum disorders, developmental coordination disorder, and/or attention deficit hyperactivity disorder. Journal of the International Neuropsychological Society, 13(2), 246-256.

Duquette MM., Carbonneau H., Roult, R., Crevier L. (2016). Sport and physical activity: Facilitating interventions with young people living with an autism spectrum disorder. Physical Activity Review, 4, 40-49.

Downey, R., \& Rapport, M. J. K. (2012). Motor activity in children with autism: A review of current literature. Pediatric Physical Therapy, 24(1), 2-20.

Duquette, M.-M., Carbonneau, H., Roult, R., \& Crevier, L. (2016). Sport and physical activity: Facilitating interventions with young people living with an autism spectrum disorder. Physical Activity Review, 4(5), 40-49.

Jansiewicz, E. M., Goldberg, M. C., Newschaffer, C. J., Denckla, M. B., Landa, R., \& Mostofsky, S. H. (2006). Motor signs distinguish children with high functioning autism and Asperger's syndrome from controls. Journal of Autism and Developmental Disorders, 36(5), 613-621.

Krakowiak, P., Goodlin-Jones, B., Hertz-Picciotto, I., Croen, LA., Hansen, RL. (2008). Sleep problems in children with autism spectrum disorders, developmental delays, and typical development: A population-based study. Journal of Sleep Research, 17, 197-206.

Kuhlthau, K., Orlich, F., Hall, T. A., Sikora, D., Kovacs, E. A., Delahaye, J., \& Clemons, T. E. (2010). Health-Related Quality of Life in children with autism spectrum disorders: results from the autism treatment network. Journal of Autism and Developmental Disorders, 40(6), 721-729.

Law, B., Bruner, B., Scharoun Benson, S. M., Anderson, K., Gregg, M., Hall, N., Tremblay, M. S. (2018). Associations between teacher training and measures of physical literacy among Canadian 8- to 12-year-old students. BMC Public Health, 18(Suppl 2).

Lincoln, A. J., Allen, M. H., \& Kilman, A. (1995). The Assessment and Interpretation of Intellectual Abilities in People with Autism. Learning and Cognition in Autism, 89-117.

Liu, X., Hubbard, J. A., Fabes, R. A., \& Adam, J. B. (2006). Sleep disturbances and correlates of children with autism spectrum disorders. Child Psychiatry and Human Development, 37(2), 179-191.

Longmuir, P. E., Boyer, C., Lloyd, M., Borghese, M. M., Knight, E., Saunders, T. J., Tremblay, M. S. (2017). Canadian Agility and Movement Skill Assessment (CAMSA): Validity, objectivity, and reliability evidence for children 8-12 years of age. Journal of Sport and Health Science, 6(2), 231-240. 
MacDonald, M., Esposito, P., Ulrich, D. (2011). The physical activity patterns of children with autism. BMC Research Notes, 4, 422.

Manjiviona, J., \& Prior, M. (1995). Comparison of Asperger syndrome and high-functioning autistic children on a Test of Motor Impairment. Journal of Autism and Developmental Disorders, 25(1), 23-39.

Memisevic, H., \& M, D. (2019). Quality of Life. In Matson J (Ed.), Handbook of Intellectual Disabilities. Autism and Child Psychopathology. Springer.

Newschaffer, C. J., Croen, L. A., Daniels, J., Giarelli, E., Grether, J. K., Levy, S. E., Windham, G. C. (2007). The Epidemiology of Autism Spectrum Disorders. Annual Review of Public Health, 28(1), 235-258.

Orr, K., Wright, F. V., Grassmann, V., McPherson, A. C., Faulkner, G. E., \& Arbour-Nicitopoulos, K. P. (2019). Children and youth with impairments in social skills and cognition in out-of-school time inclusive physical activity programs: a scoping review. International Journal of Developmental Disabilities, 14(1), 79-93.

Ortenburger, D., Rodziewicz-Gruhn, J., Wąsik, J., Marfina, O., Polina, N. (2017). Selected problems of the relation between pain-immunityand depression, Physical Activity Review, 5, 74-77.

Ozonoff, S., Young, G. S., Steinfeld, M. B., Hill, M. M., Cook, I., Hutman, T., Sigman, M. (2009). How early do parent concerns predict later autism diagnosis? Journal of Developmental and Behavioral Pediatrics, 30(5), 367-375

Pace, M., \& Bricout, V. A. (2015). Low heart rate response of children with autism spectrum disorders in comparison to controls during physical exercise. Physiology and Behavior, 141, 63-68.

Pan, C. Y. (2014). Motor proficiency and physical fitness in adolescent males with and without autism spectrum disorders. Autism, 18(2), 156-165.

Paula, I. (2018). Differential diagnosis between obsessive compulsive disorder and restrictive and repetitive behavioural patterns. Revista de Psiquiatría y Salud Mental, 6, 178-186.

Pavlova, I., Bodnar, I., \& Vitos, J. (2018). The role of karate in preparing boys for school education. Physical Activity Review. 6 , 54-63.

Persico, A. M., \& Bourgeron, T. (2006). Searching for ways out of the autism maze: genetic, epigenetic and environmental clues. Trends in Neurosciences, 29(7), 349-358.

Rinne, M. B., Pasanen, M. E., Milunpalo, S. I., \& Oja, P. (2001). Test-retest reproducibility and inter-rater reliability of a motor skill test battery for adults. International Journal of Sports Medicine, 22, 192-200.

Rooks, D. S., Kiel, D. P., Parsons, C., \& Hayes, W. C. (1997). Self-paced resistance training and walking exercise in communitydwelling older adults: Effects on neuromotor performance. Journals of Gerontology - Series A Biological Sciences and Medical Sciences, 52(3).

Sahin, M., \& Sur, M. (2015). Genes, circuits, and precision therapies for autism and related neurodevelopmental disorders. Science, 350(6263).

Sigmundsson, H., Lorås, H., \& Haga, M. (2016). Assessment of Motor Competence Across the Life Span: Aspects of Reliability and Validity of a New Test Battery. SAGE Open, 6(1).

Staples, K. L., \& Reid, G. (2010). Fundamental movement skills and autism spectrum disorders. Journal of Autism and Developmental Disorders, 40(2), 209-217.

Taira, M., Takase, Miki., Sasaki, H. (1998). Sleep disorder in children with autism. Psychiatry and Clinical Neuroscience, 52, 182-183.

Tyler, K., MacDonald, M., \& Menear, K. (2014). Physical Activity and Physical Fitness of School-Aged Children and Youth with Autism Spectrum Disorders. Autism Research and Treatment, 2014, 1-6.

Van Waelvelde, H., Oostra, A., Dewitte, G., Van Den Broeck, C., \& Jongmans, M. J. (2010). Stability of motor problems in young children with or at risk of autism spectrum disorders, ADHD, and or developmental coordination disorder. Developmental Medicine and Child Neurology, 52(8).

Varni, J. W., Seid, M., \& Rode, C. A. (1999). The PedsQL: measurement model for the pediatric quality of life inventory. Medical Care, 37(2), 126-139.

Varni, James W., Limbers, C. A., \& Burwinkle, T. M. (2007). Impaired health-related quality of life in children and adolescents with chronic conditions: A comparative analysis of 10 disease clusters and 33 disease categories/severities utilizing the PedsQLTM 4.0 Generic Core Scales. Health and Quality of Life Outcomes, 5.

Wei, X., Wagner, M., Christiano, E. R. A., Shattuck, P., \& Yu, J. W. (2014). Special Education Services Received by Students With Autism Spectrum Disorders From Preschool Through High School. Journal of Special Education, 48(3), 167-179.

Whitehead, M. (2010). The concept of physical literacy. Physical Literacy. European Journal of Physical Education, 6(2), $127-138$.

Zanevsky, I. P., \& Zanevska, L. H. (2013). Ruffier test model taking into account an age of the patient. Teorija Ta Metodyka Fizychnogo Vyhovannja, 2, 17-27. 